\title{
Morphology and Clinical Presentation of Duplicate External Auditory Canal-Literature Review
}

\author{
Shuaib Kayode AREMU ${ }^{1}$ Tayo IBRAHIM ${ }^{2} \quad$ Kayode Rasaq ADEWOYE ${ }^{3}$ \\ Adewale Daniel AGBAAKIN ${ }^{4}$ \\ 1.Department of Otorhinolaryngology, Federal Teaching Hospital Ido-Ekiti/Afe Babalola University, \\ Ado-Ekiti,Ekiti State,Nigeria \\ 2. Department of Ophthalmology, Federal Teaching Hospital Ido-Ekiti/Afe Babalola University, Ado-Ekiti,Ekiti \\ State,Nigeria \\ 3. Department of Community Medicine, Federal Teaching Hospital Ido-Ekiti/Afe Babalola University, Ado- \\ Ekiti,Ekiti State,Nigeria \\ 4. Department of Otorhinolaryngology, Federal Teaching Hospital Ido-Ekiti,Ekiti State,Nigeria
}

\begin{abstract}
First branchial cleft anomalies (FBCA) such as duplication of the external auditory canal are due to failure to obliterate the ventral portion of the 1st cleft while external auditory canal stenosis and atresia are abnormalities of the dorsal portion. The annual incidence is 1:1000,000 and it's been found to be commoner among females $69 \%$ than males $31 \%$ with the left ear most affected.FBCA can present as fistula, sinus or cysts located around/along the EAC, periauricular region and the neck above the hyoid bone.

Keywords: Duplicate External auditory canal,First branchial anomaly,auditory canal stenosis

DOI: $10.7176 / \mathrm{JHMN} / 64-04$

Publication date:July $31^{\text {st }} 2019$

\section{Introduction}

External Auditory canal arises from of the first Branchial arch groove which deepens and meets the Tubotympanic recess of the Endoderm with the Mesoderm contributing from the sides. The plate of tissue then formed a Meatal plug which is recanalize thus giving rise to the External Auditory canal. This in-utero recanalization process has a variable outcome and may result to a variation in the lumen of the External Auditory canal.
\end{abstract}

\section{Embryology of external auditory canal}

In the 3rd week of embryonic life, the trilaminar flat embryo begin to fold on itself and by the 4th week, five(5) bilateral swellings are seen growing cranio-caudally separated by clefts externally \& pouches internally. The whole set up is referred to as the branchial apparatus (Sadler, 2011).

The swellings are of mesoderm origin and are well branchial arches. These arches are separated from each other externally by ectoderm lined branchial cleft and internally by endoderm lined branchial pouches. Together, these structures develop into the structures of face, neck, and phanynx (Sadler, 2011; Schoenwolf et al., 2009).

The 1st branchial cleft has two portions; dorsal and ventral. The dorsal portion form the external auditory canal and cavum concha while the ventral portion obliterates by the 7 th week as the 1 st and 2nd branchial arch fuse (Schoenwolf et al., 2009).

First branchial cleft anomalies (FBCA) such as duplication of the external auditory canal are due to failure to obliterate the ventral portion of the $1 \mathrm{st}$ cleft while external auditory canal stenosis and atresia are abnormalities of the dorsal portion (Ertas et al., 2015).

There is an unpredictable relationship between parotid gland, facial nerve and persistent ventral portion of the 1st branchial cleft. This relationship is important during surgical management of FBCA (Bajaj et al., 2011). Hyndman and light reported the first case of FBCA (Hyndman \& Light, 1929; Koeller et al., 1999)

\section{Epidemiology}

Thyroglossal duct remnants are the commonest congenital head and neck lesion followed by branchial cleft anomalies which account for $20 \%$ of cases (Bajaj et al., 2011). 2nd branchial cleft anomalies, however, are the commonest cause of branchial clefts defects at $95 \%$ while 1 st branchial cleft anomalies (FBCA) account for 1$4 \%$ with 3rd \& 4th cleft being very rare (Bajaj et al., 2011). Some literature put the prevalence of FBCA at $1-8 \%$ (Bajaj et al., 2011).

The annual incidence of FBCA is 1:1000,000 (Liu et al., 2012) and it's been found to be commoner among females $69 \%$ than males $31 \%$ with the left ear most affected (Souza et al., 2002).

4.Pathological \& anatomical classification of fbca

Chandler et all categorizes the theories for the formation of FBCA into four (4) which are; persistent/incomplete 
obliteration of the branchial cleft, the persistence of vestiges of the pre-cervical sinus, the thymophanyngeal theory and cervical lymph node theory (Chandler \& Mitchell, 1981). The most widely accepted theory is that of incomplete obliteration of branchial clefts.

The first attempt at classifying FBCA was made by Arnot in 1971 (Tham \& Low, 2005). He described two morphological types;

Type I: Include a painful cyst or discharging sinus in the area of the partial gland which is often closely related with the lower branches of the facial nerve and may extend to the infratemporal fossa.

Type II: Include sinus or superficial cyst in the anterior triangle of the neck which may have an external opening below the angle of mandible, and a tract which extends to/or communicate with EAC ( Work, 1972).

Work (1972) also describes two forms of FBCA but based his classification on the tissue of origin.

Type I: Are cysts of ectoderm origin. They classically appear media to the concha and frequently extend to the postauricular crease, superior to the facial nerve. Histologically, they have a squamous lining.

Type II: Are cysts, and sinuses of both ectoderm and mesoderm origin considered also like a duplicate of EAC \& pinna. They present as abscess below the angle of mandible, with track running superiorly to the EAC with variable relationship to the facial nerve. Histologically, they contain cartilages and squamous epithelium.

Finally, Olsen et al. (1980) proposed a simpler classification based on the clinical presentation of the lesion. as; cyst, sinus or fistula.

However, the above classifications bear no clinically relevant because of the following reasons (McMurran et al., 2017)

- Lesions may not fit the descriptions easily

- Anomalies are difficult to categories based on clinical examination and imaging result

- Work classification is histologic classification which can only be done retrospectively after excision.

- The information gotten from the classifications has no bearing on the management plan.

Therefore, McMurran et al. (2017) proposed that instead of using Arnot, Work or Olsen's classification, clinicians should focus on using information gathered from clinical examination and preoperative imaging, preferably MRI to plan for surgery. Nevertheless, the relationship of the lesion to facial nerve would still remain unknown till during surgery.

Hence, McMurran et al, recommend that future reports of FBCA cases should include the relationship of the lesion to the facial nerve so as to allow for a meta-analysis of comparison of similar lesions (Ertas et al., 2015).

\section{Clinical presentation}

FBCA can present as fistula, sinus or cysts located around/along the EAC, periauricular region and the neck above the hyoid bone. The lesions can present the following symptoms based on location (Goudakos et al., 2012; Shinn et al., 2015; Triglia et al., 1998).

Auricular Symptom: Otorrhea with a fairly mucopurulent or purulent discharge, otitis media and cholesteatuna (Shinn et al., 2015; Triglia et al., 1998).

Cervical Symptom: pit type depression near the angle of the mandible. Pressing the area produce a small string of clear materials which could be purulent if infected and cause submandibular adenitis (Goudakos et al., 2012).

Parotid Symptoms: Small isolated masses located either at the lower pole of the parotid region or against the mastoid region. They are usually obvious when infected (Goudakos et al., 2012; Triglia et al., 1998).

In patients with fistula, the lower opening was located between sternocleidomastoid muscle posteriorly \& angle of mandible anteriorly, hyoid bone infernally while the upper opening is in the flood of EAC at the cartilaginous part or at the junction of the bone \& cartilaginous part of EAC (Goudakos et al., 2012; Shinn et al., 2015; Triglia et al., 1998). In sinuses, the opening is in any one of these sites.

\section{Meta-analysis of case of fbca}

A meta-analysis of 158 cases extracted from 55 journals between 1923-2000 by D'souza et al. (2002) found a high female to the male preponderance of $69 \%$ to $31 \%$ respectively with the left ear slightly more affected than right $57.9 \%$ to $42.1 \%$ respectively. They identified the relationship of the lesions to facial nerve \& classify it into deep $(30 \%$ cases $)$ when lesion lies medial to the facial nerve, superficial $(57 \%)$ when the lesion is lateral and between branches $(13 \%)$ when lesion passes between branches of the facial nerve.

Fistula lesions are more likely to pass deep to facial nerve while sinuses are usually superficial.

The commonest site of the opening of the tracks is EAC but sinuses can also open in the concha, upper neck, and postauricular skin. Tracks opening in EAC are more likely to lie deep to the facial nerve (D'Souza et al., 2002).

The mean age at presentation was 17.6yrs, 8.6yrs, and 6.5yrs for superficial, deep and between branches lesions respectively. In children $<6$ months the track is more likely to pass deep to the facial nerve (D'Souza et 
al., 2002).

The complications reported are facial nerve palsy (injury during surgery) which is the commonest, recurrence (especially when incomplete dissection of parotid \& facial nerve is not done to complete excise the lesion), infections, TM perforation, canal, and keloid formation (D'Souza et al., 2002).

McMurran et al. (2017) reported 9 cases of which 8 (88.8\%) were females \& $11.2 \%$ (1) male. They found no relationship with a side of the face affected but had a case of bilateral FBCA which is neither syndromic nor familiar (McMurran et al., 2017)

Triglia et al. (1998) reported 39 cases from 1980-1996. They identified three anatomical types; fistula (11), sinus (20) and cysts (8). There is a female to a male preponderance of $69.2 \%$ to $30.8 \%$ \& left to the right location of $53.8 \%$ to $46.2 \%$ respectively. The relationship of the lesion to the facial nerve was; deep (38.9\%) Superficial $(50 \%)$ and in between branches $(11.1 \%)$.

The problems encountered are similar to those presented by D'Souza et al. (2002) but they had a higher number of cases of misdiagnosis leading to inappropriate intervention such as instillation of a sclerosing agent to the track or inadequate/superficial excision. All these interventions cause sclerosis \& recurrence and leading to the poor delineation of the facial nerve and its branches during definitive surgical excision. The result of this is facial nerve palsy (D'Souza et al., 2002; Triglia, 1998).

\section{Conclusion}

All literature reviewed agreed that the definitive treatment of FBCA is surgery. The surgery involves complete dissection of the parotid gland (parotidectomy) and facial nerve $\&$ its branches to expose the lesion track and for complete excision.

\section{References}

Sadler, T. W. (2011). Langman's medical embryology 11th edition Ch 9 (skeletal system): pp 127-134, Ch 16 (head \& neck) pp 265-287.

Schoenwolf, G., Bleyl, S., Brauer, P., Francis-West. P. (2009) Larson's uman embryology 4th edition. Churchill Livingston, New York, Edinburgh

Ertas, B., Gunaydin, R. O., \& Unal, O. F. (2015). The relationship between the fistula tract and the facial nerve in type II first branchial cleft anomalies. Auris Nasus Larynx, 42(2), 119-122.

Bajaj, Y., Ifeacho, S., Tweedie, D., Jephson, C.G., Albert, D.M., Cochrane, L.A., et al. (2011). Branchial anomalies in children. Int J Pediatr Otorhinolaryngol; 75:1020-1023.

Hyndman, O.R., Light, G. (1929). The branchial apparatus. Arch surgery. 19:410-452.

Koeller, K. K., Alamo, L., Adair, C. F., \& Smirniotopoulos, J. G. (1999). From the Archives of the AFIP: Congenital cystic masses of the neck: Radiologic-pathologic correlation. Radiographics, 19(1), 121-146. doi: 10.1148/radiographics.19.1.g99ja06121.

Liu, Y., Li, T., Xue, J., Jia, J., Xiao, S., \& Zhao, E. (2012). First branchial cleft fistula presenting with internal opening on the Eustachian tube: Illustrated cases and literature review. International journal of pediatric otorhinolaryngology, 76(5), 642-645.

D'Souza, A. R., Uppal, H. S., De, R., \& Zeitoun, H. (2002). Updating concepts of first branchial cleft defects: a literature review. International Journal of Pediatric Otorhinolaryngology, 62(2), 103-109.

Chandler, J. R., \& Mitchell, B. (1981). Branchial cleft cysts, sinuses, and fistulas. Otolaryngologic Clinics of North America, 14(1), 175.

Tham, Y. S., \& Low, W. K. (2005). First branchial cleft anomalies have relevance in otology and more. Ann Acad Med Singapore, 34(4), 335-338.

Work, W. P. (1972). Newer concepts of first branchial cleft defects. The Laryngoscope, 82(9), 1581-1593.

Olsen, K. D., Maragos, N. E., \& Weiland, L. H. (1980). First branchial cleft anomalies. The Laryngoscope, 90(3), 423-436.

McMurran, A. E., Clement, W. A., Davis, C. F., Irwin, G. J., Wynne, D. M., \& Kubba, H. (2017). Classification of first branchial cleft anomalies: is it clinically relevant?. Annals of Pediatric Surgery, 13(1), 8-13. Doi: http://dx.doi.org/10.1097/01.XPS.0000459983.88215.34

Goudakos, J. K., Blioskas, S., Psillas, G., Vital, V., \& Markou, K. (2012). Duplication of the external auditory canal: two cases and a review of the literature. Case reports in otolaryngology, 2012. Doi http://dx.doi.org/10.1155/2012/924571

Shinn, J. R., Purcell, P. L., Horn, D. L., Sie, K. C., \& Manning, S. C. (2015). First branchial cleft anomalies: otologic manifestations and treatment outcomes. Otolaryngology-head and Neck Surgery, 152(3), 506-512. doi:10.1177/0194599814562773.

Triglia, J. M., Nicollas, R., Ducroz, V., Koltai, P. J., \& Garabedian, E. N. (1998). First branchial cleft anomalies: a study of 39 cases and a review of the literature. Archives of Otolaryngology-Head \& Neck Surgery, 124(3), 291-295. 
9. Acknowledgements

I appreciate all the members of my family for their supports

10. Conflicts Of Interest Disclosure

There is no conflict of interest with anyone 